\title{
Deep Neural Artificial Intelligence for IoT Based Tele Health Data Analytics
}

\author{
Nithya Rekha Sivakumar ${ }^{1, *}$ and Ahmed Zohair Ibrahim ${ }^{2}$ \\ ${ }^{1}$ Department of Computer Sciences, College of Computer and Information Sciences, Princess Nourah Bint Abdulrahman \\ University, Riyadh, 11671, Saudi Arabia \\ ${ }^{2}$ Department of Computer Sciences, College of Computer and Information Sciences, Princess Nourah Bint Abdulrahman \\ University, Riyadh, 11671, Saudi Arabia \\ *Corresponding Author: Nithya Rekha Sivakumar. Email: NRRaveendiran@pnu.edu.sa \\ Received: 30 March 2021; Accepted: 01 May 2021
}

\begin{abstract}
Tele health utilizes information and communication mechanisms to convey medical information for providing clinical and educational assistances. It makes an effort to get the better of issues of health service delivery involving time factor, space and laborious terrains, validating cost-efficiency and finer ingress in both developed and developing countries. Tele health has been categorized into either real-time electronic communication, or store-andforward communication. In recent years, a third-class has been perceived as remote healthcare monitoring or tele health, presuming data obtained via Internet of Things (IOT). Although, tele health data analytics and machine learning have been researched in great depth, there is a dearth of studies that entirely concentrate on the progress of ML-based techniques for tele health data analytics in the IoT healthcare sector. Motivated by this fact, in this work a method called, Weighted Bayesian and Polynomial Taylor Deep Network (WB-PTDN) is proposed to improve health prediction in a computationally efficient and accurate manner. First, the Independent Component Data Arrangement model is designed with the objective of normalizing the data obtained from the Physionet dataset. Next, with the normalized data as input, Weighted Bayesian Feature Extraction is applied to minimize the dimensionality involved and therefore extracting the relevant features for further health risk analysis. Finally, to obtain reliable predictions concerning tele health data analytics, First Order Polynomial Taylor DNN-based Feature Homogenization is proposed that with the aid of First Order Polynomial Taylor function updates the new results based on the result analysis of old values and therefore provides increased transparency in decision making. The comparison of proposed and existing methods indicates that the WB-PTDN method achieves higher accuracy, true positive rate and lesser response time for IoT based tele health data analytics than the traditional methods.
\end{abstract}

Keywords: Telehealth; internet of things; independent component; weighted bayesian; first order polynomial; taylor; deep neural network 


\section{Introduction}

With the assistance of the Internet of Things (IoT), every connected node are said to communicate with each other nodes with the objective of transferring indispensable data for precise and instantaneous decision making. This makes IoT a very efficient environment in critical circumstances like medical healthcare systems. IoT can significantly bestow platform for emerging smart healthcare systems. The transformation of traditional healthcare systems to a data driven and patient-centric healthcare has instituted a realistic revamping in the health demographics. This adaptation has minimized the casualty estimate of populations and has surged average life expectancy.

Machine learning-based healthcare monitoring framework was proposed in [1] with the purpose of consistently inspecting student essential indications and recognize biological and behavioral changes via smart healthcare mechanics. In this framework, the essential data were initially obtained by means of IoT devices. Followed by which machine learning method, support vector machine was utilized as a means of data analysis with which the anticipated risks of student's physiological and behavioral aspects were carried out.

The experiments conducted on the dataset disclosed the highest accuracy in detecting students' condition. Despite improvement observed in terms of accuracy, the response time involved in healthcare monitoring was not focused. To address this issue, in this work, an Independent Component Data Arrangement model along with the Weighted Bayesian Feature Extraction is proposed that in turn returning computational efficient robust features also reduces the response time involved in healthcare monitoring.

Healthcare 4.0 patterns focus at recognition of data-driven and patient-centric health systems wherein advanced sensors are said to be positioned to bestow personalized assistance. Motivated from this fact, a Deep Learning-based internet of healthcare framework for the assistance of Alzheimer patients called (DeTrAs) was proposed in [2]. The objective of the method, DeTrAs was achieved via three different phases. First, with the aid of the sensory movement data, recurrent neural network was applied to predict the Alzheimer patient. Followed by which an ensemble method employing convolutional neural network and timestamp window for detecting emotion with which the abnormality tracking for Alzheimer patients were made in a significant manner.

Finally, an IoT-based assistance mechanism was also provided for the Alzheimer patients. With this accuracy with which the prediction was made was found to be high upon comparison with the different existing machine learning algorithms. Despite improvement observed in the prediction accuracy, the reliability factor (i.e., the true positive rate) was not focused. To concentrate on the reliability of healthcare framework, in this work, First Order Polynomial Taylor DNNbased Feature Homogenization is utilized that with the aid of derivative of Polynomial Taylor ensures efficient patient-specific analysis without neglecting the former knowledge of the patient, thus increasing the overall performance of healthcare monitoring.

The contributions of Weighted Bayesian and Polynomial Taylor Deep Network (WB-PTDN) are listed below.

- To minimize the response time involved in healthcare monitoring in remote health monitoring system, a WB-PTDN method is introduced based on Deep Learning.

- An Independent Component Data Arrangement model is applied to the raw dataset so that normalization is performed in a computationally efficient manner.

- To improve the reliability of the overall decision making process using Weighted Bayesian Feature Extraction algorithm. 
- To enhance accuracy by means of First Order Polynomial Taylor DNN-based health data analytics algorithm.

- The extensive simulation is carried out to measure the performance of the WB-PTDN method and other related methods. The simulation result illustrates that our WB-PTDN method provides improved performance.

The rest of the work is organized as follows. In Section 2 related works are covered and Section enumerates the methodology followed to carry out this research work and discusses in detail the evaluation algorithms that are used to validate the proposed method. Section 4 covers the experimentation details, followed by in depth discussion based on the experimental results in Section 5, finally Section 6 presents concluding remarks.

\section{Related Works}

In this section, we present contemporary pertinent related works to our work that led and motivated us to carry out this research work. The related works are related to machine learning and deep learning methods along with the review of works conducted in the area of remote monitoring system. In recent few years, the healthcare industry has overlooked notable coercion owing to the ceaseless upsurge in patients concerning chronic diseases and their long-term treatment given to the patient. Different clinical applications have been proposed to provide solutions to the surge rate of chronic patients in hospital.

An integrative review of methods was investigated in [3]. Mental health remains to be an essential part in ensuring quality of life. Presently, there is an absence of an unbiased strategy for mental health assessment and also almost available methods are constrained to face-to-face discussions between patient and doctor. In [4] an integration of convolutional neural network and long short-term memory (CNN-LSTM) algorithms analyzing the visual metrics was proposed.

People suffering from chronic disease are said to be increasing in a rapid manner, therefore pushing the healthcare industry more demanding issues. With this objective, there exist numerous ontology and IoT-based healthcare systems to logically administer the chronic patients for longterm care. In [5], type-2 fuzzy ontology-aided recommendation systems for IoT-based healthcare was proposed with the purpose of significantly keeping an eye on the patient's body while recommending diets for analyzing patient risk factors. A global survey on machine learning system for IoT-enabled smart health care was proposed in [6]. The emergence of Internet of Things (IoT) technologies smoothen the advancement of healthcare from face-to-face discussion to tele health data analytics. A smart healthcare system in IoT environment was designed in [7] with the objective of monitoring a patient's fundamental health indications and the room condition where the patients have to be in real-time.

The IoT bestows suitable mechanisms for a broad range of applications including smart cities, traffic maintenance, waste management, emergency services health care and so on. Amongst them, one of the most fascinating applications for the IoT is medical care and health care. An ensemble RNN deep neural network was applied in [8,9] to monitor health risk. Yet another comprehensive survey on the Internet of Things for health care was investigated in [10].

The unparalleled edge of IoT and various scopes of prospective IoT applications are arousing its patients to force in any domain, to name a few being, healthcare, catastrophe reaction to instinctive and man-made mishaps notifying and rehabilitation system. A systematic literature review on health care applications was proposed in [11]. Yet another systematic mapping study 
from software engineering view point was presented in [12]. A comprehensive review on distributed healthcare for internet of things was designed in [13].

In recent years, with an ever mounting spreading of medical sensors and Internet of things devices that are laboriously switching the way healthcare is addressed globally. In this purview, an integration of Cloud and IoT architectures envisage healthcare systems potential of reinforcing real time applications when performing Artificial Intelligence on data fabricated by sensors. However, the response time together with security and privacy, poses critical issues. To address this issue, in [14], a comprehensive review regarding the utilization of IoT in health care was proposed. A machine learning technique called convoltuoinal neural network was applied in [15] for disease prediction using structured and unstructured data. Yet another mechanism for disease classification using deep and machine learning was proposed in [16].

A cognitive healthcare framework applying both the Internet of Things (IoT) and cloud environment utilizing smart sensors for communications and intelligent decision making using deep was proposed in [17]. Yet another method to ensure low cost solution for IoT based healthcare system using fuzzy neural network was presented in [18]. One of the mechanisms becoming most eminent is the feature selection, specifically in the data sets consisting of numerous variables and features. With appropriate feature selection irrelevant variables are said to be discarded, therefore improving the classification accuracy. Machine learning method was applied in [19] for enhancing the accuracy involved in classification.

In light of the above discussion on the existing methods, it is comprehensible that there is a research gap in the area of IoT based tele health data analytics. Most of these systems are based on classic machine learning and deep learning techniques, which are unable to find patient health risk in a precise and timely manner and provide decision making accordingly. The classic machine learning and deep learning techniques cannot achieve the necessary health risk analysis in a timely manner with minimum response time. In addition, a deep learning based internet of healthcare framework detects abnormality tracking to a limited extent, and cannot perfectly address the reliability issue. Therefore, the proposed Weighted Bayesian and Polynomial Taylor Deep Network (WB-PTDN) method is a novel effort to design an intelligent process for IoT based tele health data analytics.

\section{Weighted Bayesian and Polynomial Taylor Deep Network (WB-PTDN) for IoT-Based Tele Health Data Analytics}

In recent years, there has been an expeditious and epidemic surge in the volume of healthassociated digital data that is initiated by the citizens themselves and also the healthcare providers. Also speaking in a global manner, there has been an extensive surge in chronic disease. This integrated with an increasing aging populations have resulted in the evolution of tele health that provides promising solutions for remote healthcare diagnosis, monitoring, and so on. Motivated by this fact, in this work, a multilayered architecture is utilized in deep learning with the objective of mapping the relations between inputs features (i.e., data obtained from each patient) and the outcomes (i.e., resultant healthcare analysis). This architecture makes deep learning more appropriate for inspecting numerous variables (i.e., columns in training data file, i.e., vital signs, laboratory values, demographics).

The framework carrying several levels of nonlinearities shines at recognizing complicated data patterns. However, in a remote healthcare monitoring system, data that comes from numerous sources required to be equipped into multiple models. The integration of the framework is hence 
said to performed at a specific level to attain the overall learning objective (i.e., computationally efficient and precise IoT-based tele health data analytics). Fig. 1 given below shows the block diagram of Weighted Bayesian and Polynomial Taylor Deep Network (WB-PTDN) for IoT-based Tele Health Data Analytics.

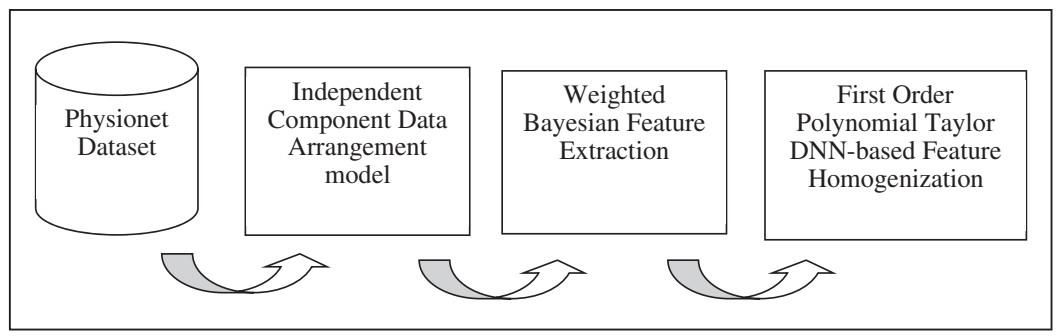

Figure 1: Block diagram of WB-PTDN for IoT-based tele health data analytics

As shown in the above figure, the proposed WB-PTDN method involves three different stages. To start with the Physionet dataset provided as input, computationally efficient data normalization is performed by means of Independent Component Data Arrangement model. With the normalized data, dimensionality reduced features are obtained by utilizing Weighted Bayesian Feature Extraction model. Finally, with the extracted data, First Order Polynomial Taylor DNN-based for IoT based tele health data analytics is presented. To start with a system model for remote health monitoring for IoT based tele health data analytics is designed. Followed by which the description of each model is presented in detail.

\subsection{Remote Healthcare System Model}

In this section, a Remote Healthcare System model for IoT based tele health data analytics is designed. This system model forms the basis for obtaining the overall objective of health analysis in a timely and precise manner. Fig. 2 given below shows the Remote Healthcare System model utilized in our work. It consists of three different layers.

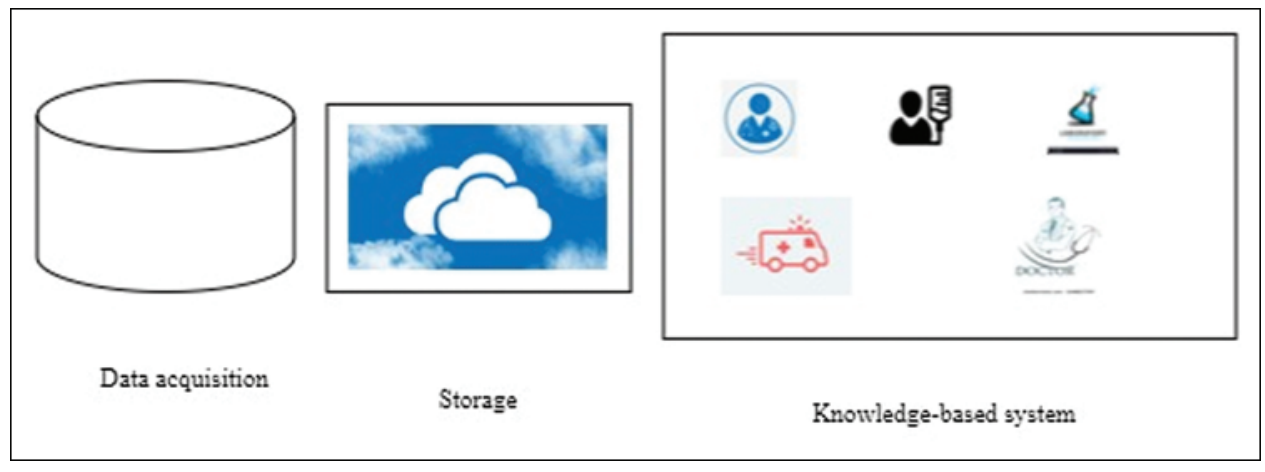

Figure 2: Remote healthcare system model

As shown in Fig. 2. The remote healthcare monitoring system of tele health data analytics utilized in our work comprises of data acquisition, storage and the knowledge-based system. First, data are acquired from the Physionet dataset. Here, with the aid of numerous sensors 
' $S=S_{1}, S_{2}, \ldots, S_{n}$ ', details about patients health data are obtained. With the obtained data ' $D=D_{1}, D_{2}, \ldots, D_{n}$ ', to enhance the quality of service, data are stored in the cloud environment. This is mathematically expressed as given below.

$$
\text { RHMS }=\left|\begin{array}{cccc}
S_{1} D_{1} & S_{1} D_{2} & \ldots & S_{1} D_{n} \\
S_{2} D_{1} & S_{2} D_{2} & \ldots & S_{2} D_{n} \\
\ldots & \ldots & \ldots & \ldots \\
S_{n} D_{1} & S_{n} D_{2} & \ldots & S_{n} D_{n}
\end{array}\right|
$$

Here, with the assistance of servers or virtual machines ' $V M=V M_{1}, V M_{2}, \ldots, V M_{n}$ ', the patient's status is managed that in turn improves the overall system efficiency. This is mathematically formulated as given below.

$$
V M_{1} \rightarrow\left(S_{1} D_{1}: S_{1} D_{2}: S_{1} D_{n}\right), V M_{2} \rightarrow\left(S_{2} D_{1}: S_{2} D_{2}: S_{2} D_{n}\right), \ldots, V M_{n} \rightarrow\left(S_{n} D_{1}: S_{n} D_{2}: S_{n} D_{n}\right)
$$

Finally, the knowledge-based system where the actual remote healthcare monitoring is performed ensures precise decision making by obtaining the various data based on the results of physician, lab, patient, hospital, ambulance and so on.

\subsection{Independent Component Data Arrangement Model}

In this section, the data arrangement using Independent Component Analysis called, Independent Component Data Arrangement model is presented. The data arrangement for each patient is achieved by an integration node that surrounds numerous elements as shown in Fig. 3.

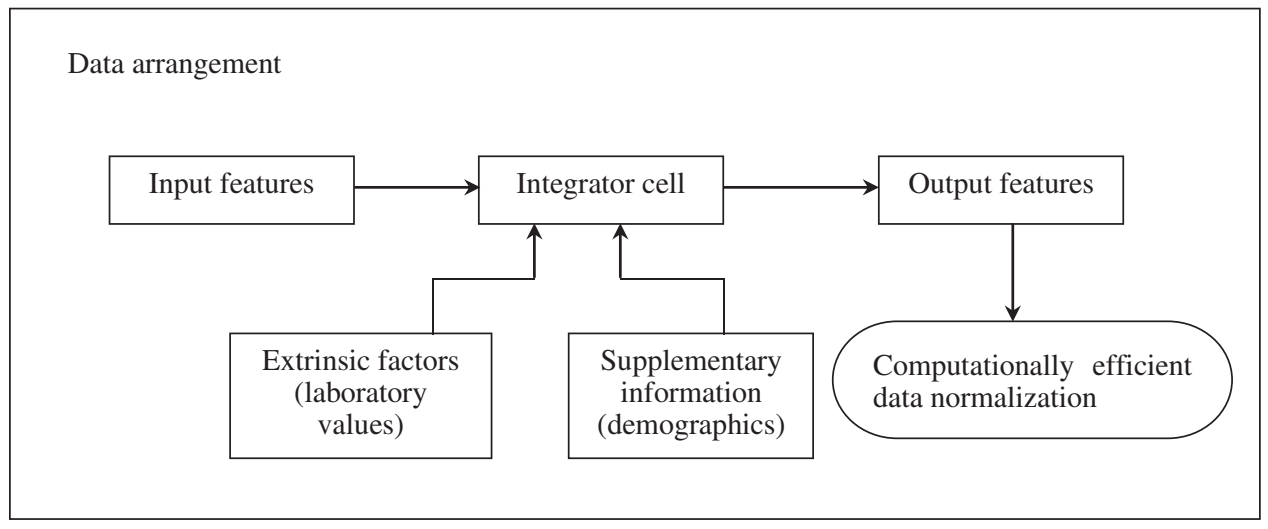

Figure 3: Block diagram of independent component data arrangement model

The input features are the original information obtained from ICU patients in three separate hospital systems. Integrator cell is the smallest element of information nitration systems. The integrator cell acts as a mediator between numerous input features from the data source and the global healthcare information processing systems. Integrator cell refines the input data elements with the constraints or indicators set in the extrinsic factors and supplementary information, therefore ensuring the quality of the output features meeting the prerequisites for supplementary integration processing.

The extrinsic factors contains (i.e., laboratory values) that can drive and guide the integration process. These laboratory values contain the information needed to process the data in definite 
formats (i.e., string, numeric, Boolean, real numbers and so on). The supplementary information caches laboratory values to aid the integration process. The results obtained from the integration processes are recorded in supplementary information to improve future integration. The process of integrator cell is specifically a classifier, and the output features represents the maps of classes and its confidence volumes. In our work, Deep Neural Network is utilized in designing the integrator cell. Here, different data types are identified by means of Independent Component Analysis (ICA). ICA being a multivariate analysis technique describes data with an orthogonal transformation of the coordinates that identifies additively separable elements instead of successive approximations.

Let us consider a 'n-dimensional' dataset ' $P=\left\{P_{i} \in i^{n} \mid i=1,2, \ldots, n\right\}$ ' can be roughly denoted by a smaller 'j-dimensional' dataset ' $Q=\left\{Q_{n} \in i^{j} \mid n=1,2, \ldots, j\right\}$ '. Here, ' $Q$ ' is said to be obtained by ' $Q E_{k}$ ', which refers to projecting ' $Q$ ' onto the eigenvectors representing the largest eigen values, with ' $E_{k}$ ' referring to the ' $E_{k}$ ' column of the eigen vectors ' $E$ '. Followed by which a feed forward network is trained to produce an optimal mapping between input features and outputs features via back-propagation. With the feed forward network possessing 'n' input features and ' $n$ ' output features and a single hidden layer with $k$ nodes. In the training phase, the ICA projection matrix for each 'RHMS' is measured and the output features obtained from ICA is fed to the deep neural network, with hyperbolic tangent as the activation function, is defined as given below.

$z=A(I D S)=1-\frac{2}{1+\exp (2 I D S)} \in$

From the above Eq. (3), 'IDS' forms the input dataset and ' $z$ ' forms the output dataset respectively. The training output features are compared with the actual output features and accordingly synapse weights are changed with the objective of minimizing the errors. Upon successful completion of the training process, the synapses weight is retained in the supplementary information of the integrator node. Upon determination of the data type of each column in the training data file, the format specification in extrinsic knowledge is said to be utilized in order to validate the identification results. Upon incorrect identification, error is said to be recorded, an error will be recorded in supplementary information to enhance future decision making process. The pseudo code representation of Deep Independent Component Analysis is given below.

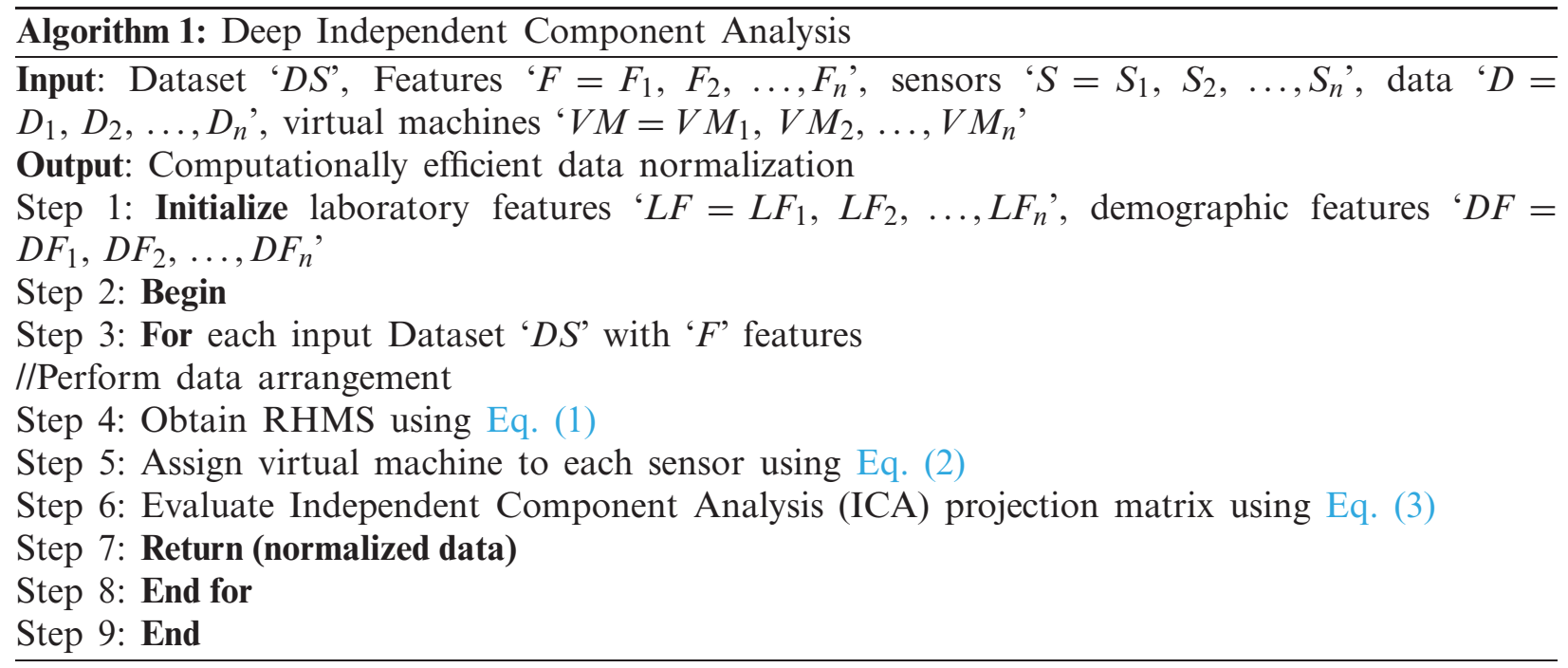


As given in the above Deep Independent Component Analysis algorithm, the objective remains in arranging the data by means of Independent Component Analysis for each Demographics, Vital Signs, and Laboratory values respectively. The proposed data arrangement processes involve arranging the information elements for each patient and reshuffling them to characterizations appropriate for facilitating analysis in a more computationally efficient manner.

\subsection{Weighted Bayesian Feature Extraction}

At the second stage of the framework, integration processes include feature extraction and feature homogenization. On one hand, the feature extraction obtains the relevant and essential features accommodating the maximal information concerning the prediction objectives in the next integration stage. With the extraction process, both size and volume of data are minimized and therefore improves the efficiency and accuracy of decision making process. An integration node for the feature extraction comprises numerous elements as illustrated in Fig. 4.

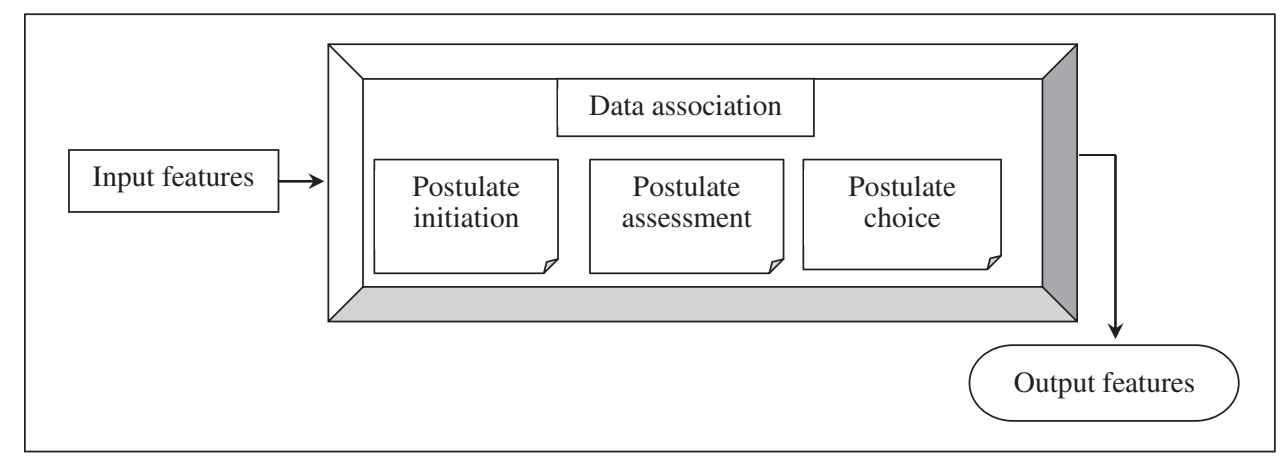

Figure 4: Weighted bayesian feature extraction

The chief task involved in the feature extraction process is Data Association. This Data Association decides which input features connect with which data elements presently. The association process is mastered by means of three functions, namely, Postulate Initiation, Postulate Assessment and Postulate Choice. With the assistance of the correlation process, the input features are said to be normalized and hence used for robust decision making.

Postulate Initiation recognizes realistic relationship candidate solutions that can be obtained from the input features, where the postulates of the relationship between the input features and the health risks will be initiated. For example, running records taken from vital signs can be utilized as the input features, and a postulate can be generated to represent a correlation between simulation runs conducted and a health risk (i.e., hearth problems, wheezing, fever and so on). Next, the postulate assessment is evaluated based on the Weighted Bayesian Confidence function. Let ' $X$ ' represents an itemset (i.e., vital signs record) comprising of items (features i.e., columns $1-8$ ) ' $I=$ $I_{1}, I_{2}, \ldots, I_{n}$ ' and let 'HR' represent the health risk associated (i.e., diagnosis connected to health risk), then, the association ' $\mathrm{X} \Rightarrow$ WHR', then, the Weighted Bayesian Confidence is measured as given below.

$$
\begin{aligned}
W B C(X \Rightarrow W H R)= & \operatorname{Prob}(H R \mid X)=\frac{\operatorname{Prob}(X, H R)}{\operatorname{Prob}(X)}=\frac{\operatorname{Prob}\left(P_{1}, P_{2}, \ldots, P_{m}, P_{m+1}, P_{m+2}, \ldots, P_{n}\right)}{\operatorname{Prob}\left(\left\{P_{1}, P_{2}, \ldots, P_{m}\right\}\right)} \\
& =\frac{\operatorname{Prob}\left(P_{1}, P_{2}, \ldots, P_{m}, P_{m+1}, P_{m+2}, \ldots, P_{n}\right)}{\operatorname{SUP}\left(\left\{P_{1}, P_{2}, \ldots, P_{m}\right\}\right)}
\end{aligned}
$$


Finally, postulate choice ascertains which outputs associations the integration system should maintain and utilize for state estimation. The preservation of the state estimation is required to evaluate the probabilities of all the events for the eventualities of numerous health issues. Let us represent the probability of a health risk as 'Prob(HR)' and the eventualities of a set of associations as ' $\left\{A_{i} \mid i=1,2, \ldots n\right\}$ '. Then, the postulate choice is formulated using the association function represented as given below.

$$
\begin{aligned}
\operatorname{Prob}( & \left.H R \mid A_{1}, A_{2}, \ldots, A_{n}\right) \\
= & \frac{\operatorname{Prob}\left(H R, A_{1}, A_{2}, \ldots, A_{n}\right)}{\operatorname{Prob}\left(A_{1}, A_{2}, \ldots, A_{n}\right)} \\
= & \frac{\operatorname{Prob}(H R) \operatorname{Prob}\left(A_{1} \mid H R\right) \operatorname{Prob}\left(A_{2} \mid \operatorname{HR}, A_{1}\right), \operatorname{Prob}\left(A_{3} \mid H R, A_{1} A_{2}\right), \ldots, \operatorname{Prob}\left(A_{n} \mid H R \ldots A_{n}\right)}{\operatorname{Prob}\left(A_{1}, A_{2}, \ldots, A_{n}\right)} \\
= & \operatorname{Prob}(H R) \frac{\prod_{i=1}^{n} \operatorname{Prob}\left(A_{i} H R, A_{i-1}^{\prime}\right)}{\operatorname{Prob}\left(A_{1}, A_{2}, \ldots, A_{n}\right)}
\end{aligned}
$$

With the above association function, the similarity probabilities for all the events in different time stamps for numerous health risks in tele health are said to be evaluated in a timely and precise manner. Each feature extraction node controls an integrated situation representation for the data from its corresponding data source. The pseudo code representation of Weighted Bayesian Feature Extraction is given below.

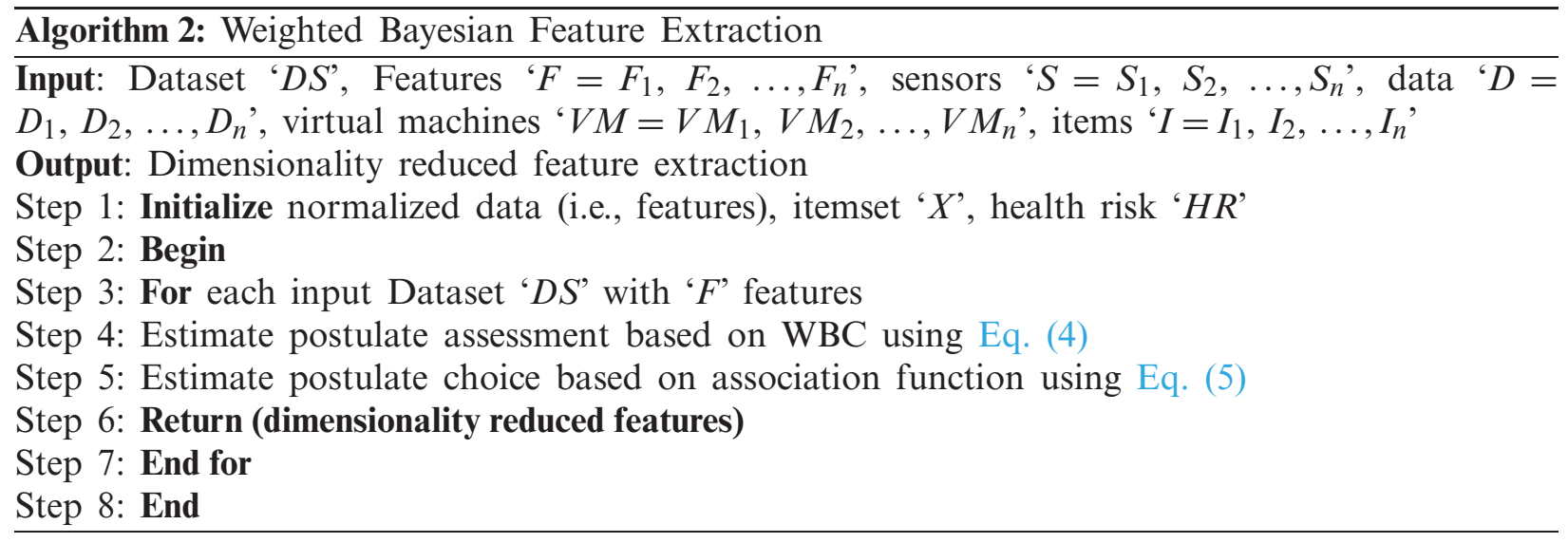

As given in the above Weighted Bayesian Feature Extraction algorithm, the objective remains in extracting the dimensionality reduced features by means of Weighted Bayesian Confidence function and association function. First, with the normalized features provided as input, Weighted Bayesian Confidence function is applied to assess the postulates. Next, an association function is applied to estimate the dimensionality reduced features.

\subsection{First Order Polynomial Taylor DNN-Based Feature Homogenization}

In this section, we aim at providing the Deep Neural Network architecture with the potentiality to adjust to new cases, helping physicians with significant patient-specific analysis and treatment, without ignoring its previous knowledge. The feature homogenization in our work is based on a novel retraining model. However, fine-tuning is not performed here whereas only select 
to retrain the DNN layers with the input data corresponding to the existing resultant value and on the other hand, the new data.

With the aid of this retraining model, as both the old data and the new information provided by specific patient are retained, personalized risk analysis is said to be ensured in an accurate manner. The local integrated situation representations for different data sources (i.e., demographics, vital signs and laboratory values) are to be homogenized into a global situation representation, so that the overall method can be utilized for decision making in the next final stage. This process, namely, feature homogenization, is achieved by First Order Polynomial Taylor DNN-based model as shown in Fig. 5.

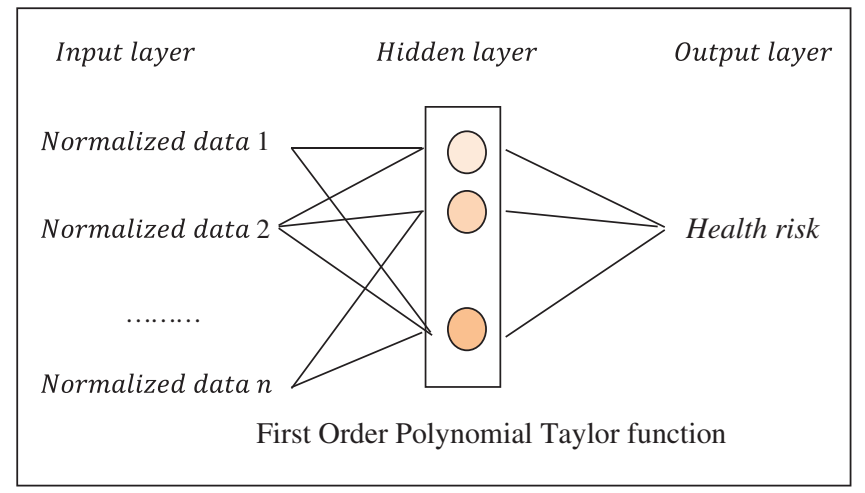

Figure 5: First order polynomial taylor DNN-based health data analytics

As shown in the above figure, let us first consider that on the basis of the deep neural network for tele health data analytics, a specific set, ' $S_{x}$ ' including the training input data corresponding to the previously estimated results and the respective patients has been formed. Let 'NS(i)' represent the network output upon application to a new sample, not included in the preceding network training data set. In addition, let ' $w_{\text {old }}$ ' represents the old weights of the Bayesian Confidence function, i.e., before retraining and let ' $w_{N e w}$ ' 'w_new' represents the new weights obtained after training. In specific ' $w_{\text {old }}^{l}$ ' and ' $w_{N e w}^{l}$ ' represents the weights associating the outputs of the last hidden layer say ' $y$ ' to the output ' $z$ '. In the proposed retraining model, the new network weights ' $w_{N e w}$ ' are evaluated by reducing the error factor as given below.

$\operatorname{Err}_{\text {new }}=\operatorname{Err}_{S_{x}, \text { new }}+\eta \operatorname{Err}_{S_{x}, \text { old }}$

From the above Eq. (6), ' $E r r_{S_{x}, \text { new }}$ ' refers to the error performed over current training set ' $S_{x}$ ', ' $E r r_{S_{x}, \text { old }}$ ' the error performed over previous training set and ' $\eta$ ' denoting the weighting factor respectively. Now, finally let us represent the variation of the network outputs (i.e., healthcare decision), after and before retraining, in the case of a Deep Neural Network (DNN), as given below.

$\Delta \operatorname{Output}_{(i)}=\operatorname{Output}_{\text {new }}(i)+\operatorname{Output}_{\text {old }}(i)$

Through First Order Taylor Polynomial and utilizing the fact that the outputs ' $z$ ' are weighted averages of the last hidden layer's outputs 'y' with the weights both new 'w_new' and old 'w_old', the new output results are estimated as given below. 
Through First Order Taylor Polynomial and utilizing the fact that the outputs ' $z$ ' are weighted averages of the last hidden layer's outputs ' $y$ ' with the weights both new ' $w_{\text {new }}$ ' and old ' $w_{\text {old }}$ ', the new output results are estimated as given below.

Output $_{\text {new }}(i)=$ Output $_{\text {old }}(i)+z_{\text {new }}^{\prime} w_{\text {new }}^{l}+w_{\text {old }}^{l}$

From the above Eq. (8) the new output value 'Output ${ }_{n e w}(i)$ ' for ' $i-t h$ ' patient is obtained on the basis of the old output value of the particular patient 'Output ${ }_{\text {old }}(i)$ ', derivative of activation function ' $z_{\text {new }}^{\prime}$ ', last new weight ' $w_{\text {new }}^{l}$ ', last old weight ' $w_{\text {old }}^{l}$ ' respectively. The pseudo code representation of First Order Polynomial Taylor DNN-based health data analytics is given below.

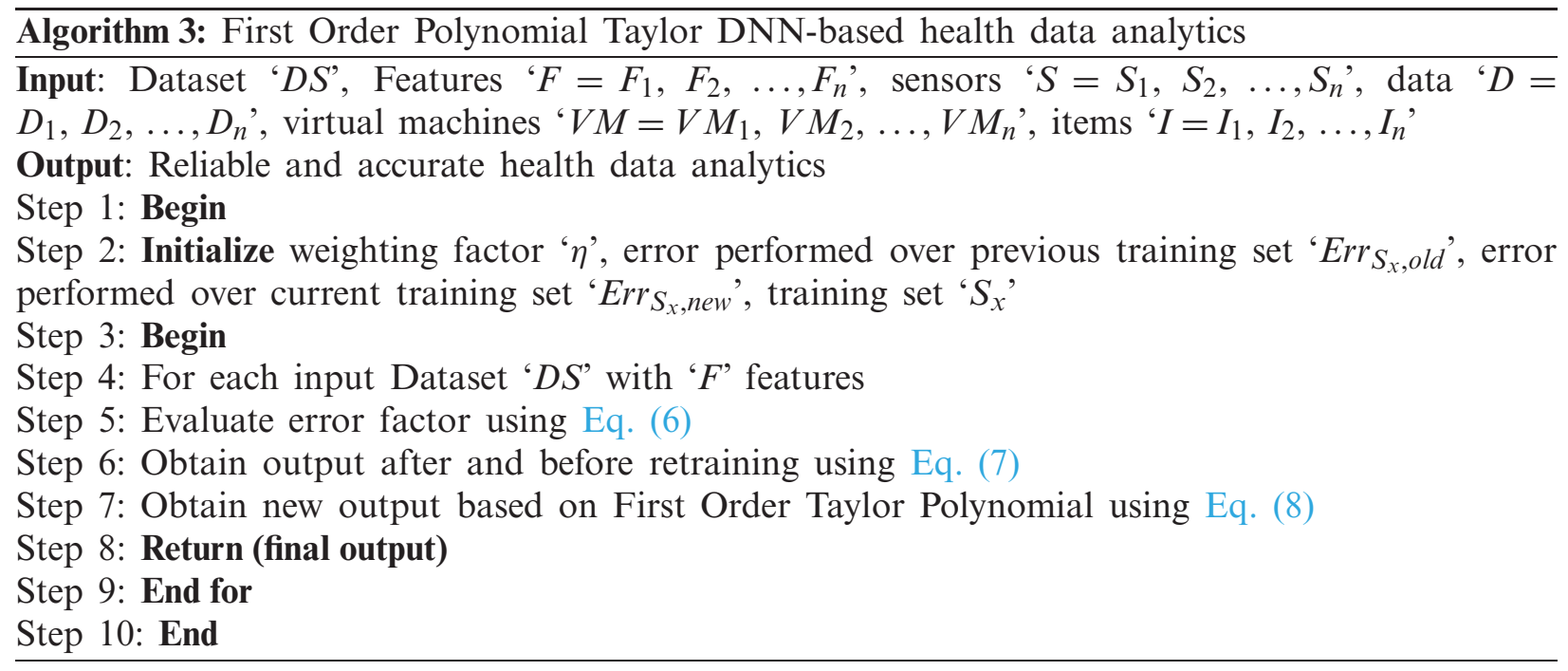

As given in the above First Order Polynomial Taylor DNN-based health data analytics algorithm, the objective remains in ensuring the reliability of the overall decision making process in addition to the accuracy of remote healthcare monitoring [20]. This is achieved in our work by first designing a retraining function by means of First Order Taylor Polynomial function. Next, the updates are made on the basis of minimization of error, therefore ensuring both reliability and accuracy for IoT-based health data analytics [21].

\section{Experimental Settings}

In this section, the simulation of the proposed Weighted Bayesian and Polynomial Taylor Deep Network (WB-PTDN) and the existing Machine learning-based healthcare monitoring framework [1] DeTrAs [2] are performed in the JAVA language and CloudSim simulator. Experiments are conducted using the Physionet dataset [22]. The dataset consists of three different tables, patient vital signs (as in Tab. 1), patient laboratory values (as in Tab. 2) and patient demographic values (as in Tab. 3). The different parameters considered for simulation is listed below. 
Table 1: Patient vital sign details

\begin{tabular}{lll}
\hline Columns & Features & Description \\
\hline 1 & HR & Heart rate \\
2 & O2Sat & Pulse oximetry \\
3 & Temp & Temperature \\
4 & SBP & Systolic BP \\
5 & MAP & Mean arterial pressure \\
6 & DBP & Diastolic BP \\
7 & Resp & Respiratory rate \\
8 & EtCO2 & End tidal carbon dioxide \\
\hline
\end{tabular}

Table 2: Patient laboratory values

\begin{tabular}{lll}
\hline Columns & Features & Description \\
\hline 9 & BaseExcess & Measure of excess bicarbonate \\
10 & $\mathrm{HCO} 3$ & Bicarbonate \\
11 & $\mathrm{FiO} 2$ & Fraction of inspired oxygen \\
12 & $\mathrm{pH}$ & N/A \\
13 & $\mathrm{PaCO} 2$ & Partial pressure of carbon dioxide from arterial blood \\
14 & $\mathrm{SaO} 2$ & Oxygen saturation from arterial blood \\
15 & $\ldots$ & $\ldots$ \\
34 & Platelets & Count \\
\hline
\end{tabular}

Table 3: Patient demographics values

\begin{tabular}{lll}
\hline Columns & Features & Description \\
\hline 35 & Age & Years \\
36 & Gender & Male/female \\
$\ldots$ & & \\
40 & ICULOS & ICU length of stay \\
\hline
\end{tabular}

\section{Results and Discussion}

The performance analysis of the proposed WB-PTDN and existing methods namely Machine learning-based healthcare monitoring framework [1] DeTrAs [2] are discussed in this section. To measure the performance, the following three metrics used are response time, reliability (in terms of true positive rate) and accuracy.

\subsection{Performance Analysis of Response Time}

The first factor or parameter to be included in remote health monitoring for IoT Based Tele Health Data Analytics is the response time. Minimum the response time, larger number of patients 
are said to be attended by the doctors and hence increasing the overall performance of healthcare monitoring. The response time is mathematically expressed as given below.

Res $_{\text {time }}=\sum_{i=1}^{n} P_{i} *$ Time $[R H M S]$

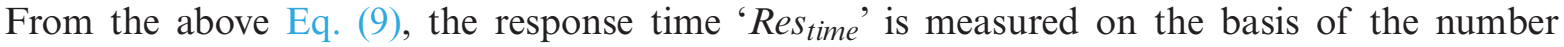
patients ' $P_{i}$ ' and the time consumed in performing the process of remote health monitoring system 'Time [RHMS]' for IoT Based Tele Health Data Analytics. It is measured in terms of milliseconds (ms).

Fig. 6 given above shows the response time involved or IoT Based Tele Health Data Analytics) using three different methods, WB-PTDN, Machine learning-based healthcare monitoring [1] and DeTrAs [2]. From the figure a direct proportionate between the numbers of patients involved in simulation and response time is observed. In other words, increasing the numbers of patients causes an increase in the analysis process and hence the overall increase in the response time is said to be observed. However, with the application of Independent Component Analysis algorithm, computationally efficient data normalization is said to be performed. This in turn arranges the information of each patient and reshuffles them to characterizations appropriate for facilitating health risk analysis, therefore minimizing the response time using WB-PTDN by $21 \%$ compared to [1] and $32 \%$ compared to [2] respectively.

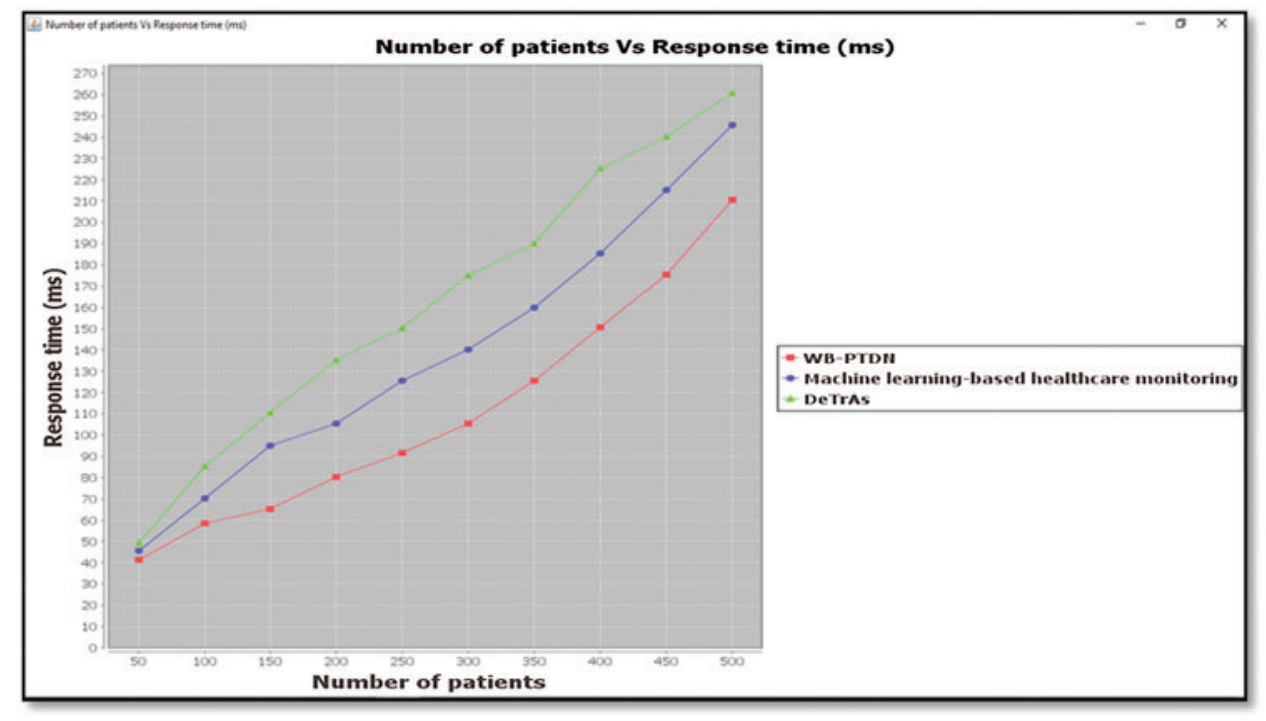

Figure 6: Graphical representation of response time

\subsection{Performance Analysis of Reliability}

The second parameter of consideration for IoT Based Tele Health Data Analytics is the reliability factor. In our work, reliability is measured on the basis of the true positive rate. Higher the true positive rate, more the reliability is said to be and hence improving the overall performance of remote health monitoring. It is measured as given below. 
$T P R=\frac{T P}{T R} * 100$

From the above Eq. (10), the true positive rate 'TPR', is measured based on the number of true positives 'TP' (i.e., health risk analyzed correctly) and the total number of risky patients 'TR' in simulation concerned. It is measured in terms of percentage (\%). Fig. 7 given above shows the reliability rate measured in terms of true positive rate. From the figure an inverse proportionate is measured between the number of patients provided for simulations and the reliability rate. In other words, increasing the numbers of patients causes a significant decrease in the reliability rate. However, simulations conducted for ' 50 ' patients involved in the process of IoT Based Tele Health Data Analytics, health risk analyzed correctly being ' 45 ' with total number of risky patients being '48' was observed to be '93.75\%' using WB-PTDN, '87.5\%' using [1] and ' $85.41 \%$ ' using [2] respectively. From the results reliability is improved using WB-PTDN upon comparison with [1] and [2]. The reason behind the improvement was due to the application of Weighted Bayesian Feature Extraction algorithm.

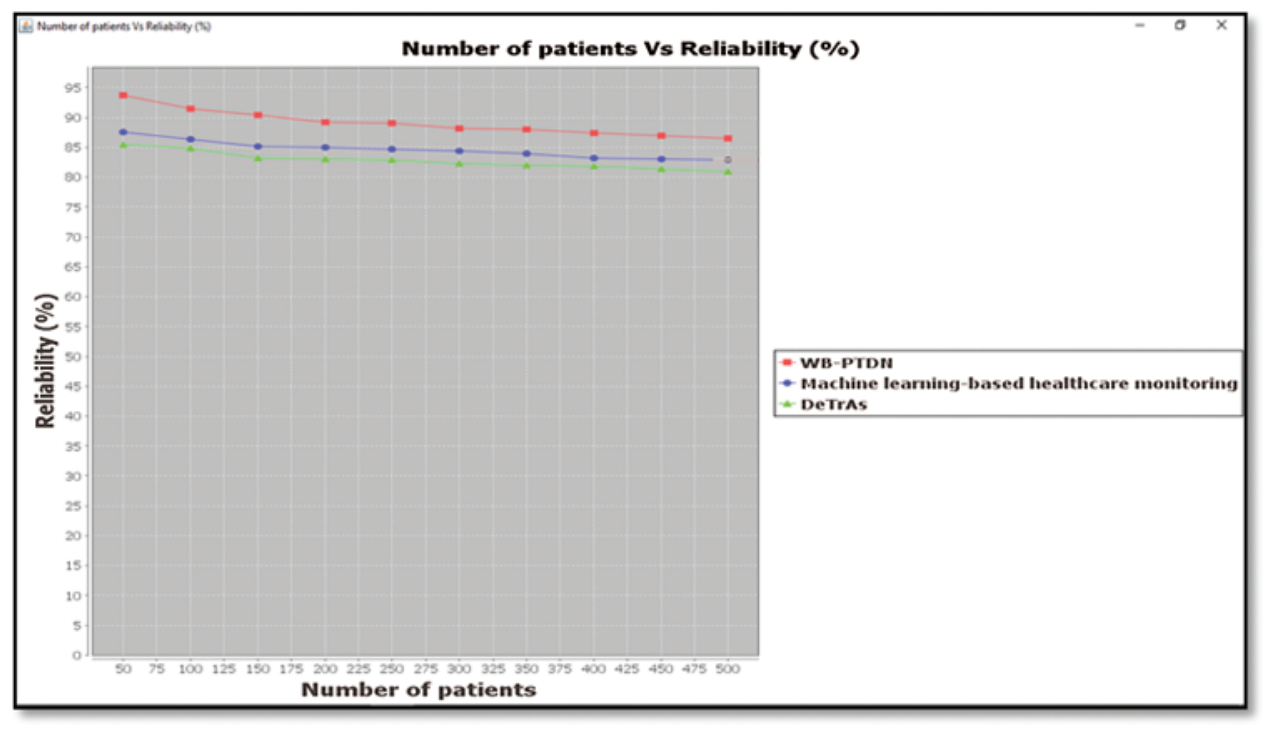

Figure 7: Graphical representation of true positive rate

By applying this algorithm, Weighted Bayesian Confidence function and association function were applied that in turn extracted dimensionality reduced features, therefore improving the reliability using WB-PTDN by 5\% compared to [1] and $8 \%$ compared to [2] respectively.

\subsection{Performance Analysis of Accuracy}

Finally, the efficiency of remote health monitoring system for IoT based tele health data analytics is measured on the basis of accuracy rate. Higher the accuracy rate more efficiency the overall performance of remote health monitoring system is said to be. This is mathematically formulated as given below.

$A c c=\frac{\sum_{i=1}^{n} \operatorname{CIHR}\left(P_{i}\right)}{\sum_{i=1}^{n}\left(P_{i}\right)} * 100$ 
From the above Eq. (11), accuracy 'Acc' is measured on the basis of the number of patients correctly identified with health risk ' $C \operatorname{IHR}\left(P_{i}\right)$ ' and the total number of patients considered for simulation ' $P_{i}$ '. It is measured in terms of percentage. Tab. 4 summarizes the accuracy obtained by the WB-PTDN, Machine learning-based healthcare monitoring [1] and DeTrAs [2].

As given in the above table, the proposed WB-PTDN is compared with state-of-the-art remote health monitoring system frameworks. WB-PTDN is found to improve the accuracy rate upon comparison with the other two existing methods, Machine learning-based healthcare monitoring [1] and DeTrAs [2] for 500 different unique patients as given in Tab. 4. However, with '50' patients involved in the experimental settings for remote health monitoring and ' $45^{\prime}$ ' patients correctly identified with health risk using WB-PTDN, '42' patients correctly identified with health risk using [1] and 41' patients correctly identified with health risk using [2], the overall accuracy was observed to be ' $90 \%$ ', ' $84 \%$ ' and ' $82 \%$ ' respectively. From the results improvement is said to be obtained using WB-PTDN upon comparison with [1] and [2] for '50' patients. The improvement was due to the application of First Order Polynomial Taylor DNN-based health data analytics algorithm. By applying this algorithm, retraining function was applied that in addition to the current status of the patient also included the previous status for measuring health risk. Also, the updates were made based on the error minimization that in turn improved the accuracy using WB-PTDN by $6 \%$ compared to [1] and $9 \%$ compared to [2] respectively.

Table 4: Tabulations for accuracy using WB-PTDN, machine learning-based healthcare monitoring [1] and DeTrAs [2]

\begin{tabular}{llll}
\hline $\begin{array}{l}\text { Number of } \\
\text { patients }\end{array}$ & Accuracy (\%) & \\
\cline { 2 - 4 } & WB-PTDN & $\begin{array}{l}\text { Machine learning-based } \\
\text { healthcare monitoring }\end{array}$ & DeTrAs \\
\hline 50 & 90 & 84 & 82 \\
100 & 88.35 & 83.55 & 81.85 \\
150 & 88.15 & 83.25 & 81.55 \\
200 & 88 & 83 & 81 \\
250 & 87.65 & 82.55 & 80.55 \\
300 & 87.4 & 82.35 & 80.35 \\
350 & 87.25 & 82 & 80 \\
400 & 87 & 81.85 & 79.55 \\
450 & 86.55 & 81.45 & 79.25 \\
500 & 86 & 81 & 79 \\
\hline
\end{tabular}

\section{Conclusion}

This paper presented a Weighted Bayesian and Polynomial Taylor Deep Network (WBPTDN) method for remote health monitoring in IoT Based Tele Health Data Analytics. In WB-PTDN, three different phases work in tandem with each other. The first phase arranges the data with the objective of normalizing the data in a computationally efficient manner on the basis of Independent Component Analysis. In phase 2, the normalized data are further subjected for dimensionality reduced feature extraction for reducing the response time in addition to the 
significant improvement in terms of reliability based on Weighted Bayesian Confidence and association function with the improved objective of analyzing health care risk analysis in the last phase. Finally, in the third stage, the analysis results are evaluated based on the First Order Polynomial Taylor DNN-based model. WB-PTDN method has been evaluated using Physionet dataset on the basis of response time, accuracy, reliability (in terms of true positive rate). The results also show that WB-PTDN method is good choice for remote health care systems and it also provides a lightweight solution in terms of its reliability and accuracy.

Acknowledgement: This Research was funded by the Deanship of Scientific Research at Princess Nourah Bint Abdulrahman University, Riyadh, Kingdom of Saudi Arabia through the Fast-track Research Funding Program.

Funding Statement: The authors received no specific funding for this study.

Conflicts of Interest: The authors declare that they have no conflicts of interest to report regarding the present study.

\section{References}

[1] A. Souri, M. Y. Ghafour, A. M. Ahmed, F. Safara, A. Yamini et al., "A new machine learning-based healthcare monitoring model for student's condition diagnosis in internet of things environment," Soft Computing, vol. 24, no. 22, pp. 17111-17121, 2020.

[2] S. Sharma, R. K. Dudeja, G. S. Aujla, R. S. Bali and N. Kumar, "Detras: Deep learning-based healthcare framework for IoT-based assistance of Alzheimer patients," Neural Computing and Applications, vol. 1, pp. 1-13, 2020. https://doi.org/10.1007/s00521-020-05327-2.

[3] E. Andrews, K. Berghofer, J. Long, A. Prescott and M. C. Stevens, "Satisfaction with the use of telehealth during COVID-19: An integrative review," International Journal of Nursing Studies Advances, vol. 2, no. 2, pp. 1-8, 2020.

[4] S. B. Shafiei, Z. Lone, A. S. Elsayed, A. A. Hussein and K. A. Guru, "Identifying mental health status using deep neural network trained by visual met," Translational Psychiatry, vol. 10, no. 1, pp. 1-8, 2020.

[5] F. Ali, P. Khan, D. Kwak, S. M. R. Islam, N. Ullah et al., "Type-2 fuzzy ontology-aided recommendation systems for IoT based healthcare," Computer Communication, vol. 119, pp. 138-155, 2018.

[6] W. Li, Y. Chai, F. Khan, S. R. Ullah, S. Verma et al., "A comprehensive survey on machine learningbased big data analytics for IoT-enabled smart healthcare system," Mobile Networks and Applications, vol. 26, pp. 234-252, 2021.

[7] M. D. M. Islam, A. Rahaman and M. D. R. Islam, "Development of smart healthcare monitoring system in IoT environment," SN Computer Science, vol. 1, pp. 1-11, 2020.

[8] M. Farsi, "Application of ensemble RNN deep neural network to the fall detection through IoT environment," Alexandria Engineering Journal, vol. 60, no. 1, pp. 199-211, 2021.

[9] J. R. Langabeer II, A. Yatsco and T. C. Langabeer, "Telehealth sustains patient engagement in OUD treatment during COVID-19," Journal of Substance Abuse Treatment, vol. 122, pp. 1-3, 2021.

[10] S. M. R. Islam, D. Kwak, M. D. H. Kabir, M. Hossain and K. S. Kwak, "The internet of things for health care: A comprehensive survey," IEEE Access, vol. 3, pp. 678-708, 2015.

[11] A. Pashazadeh and N. J. Navimipour, "Big data handling mechanisms in the healthcare applications: A comprehensive and systematic literature review," Journal of Biomedical Informatics, vol. 82, pp. 47-62, 2018.

[12] G. Marquez, H. Astudillo and C. Taramasco, "Security in telehealth systems from a software engineering viewpoint: A systematic mapping study," IEEE Access, vol. 8, pp. 10933-10950, 2020. 
[13] M. N. Birje and S. S. Hanji, "Internet of things based distributed healthcare systems: A review," Journal of Data, Information and Management, vol. 2, pp. 149-165, 2020.

[14] L. Greco, G. Percannella, P. Ritrovato, F. Tortorella and M. Vento, "Trends in IoT based solutions for health care: Moving AI to the edge," Pattern Recognition Letters, vol. 135, pp. 346-353, 2020.

[15] M. Chen, Y. Hao, K. Hwang, L. Wang and L. Wang, "Disease prediction by machine learning over big data from healthcare communities," IEEE Access, vol. 5, pp. 8869-8879, 2017.

[16] N. E. M. Khalifa, M. H. N. Taha, G. Manogaran and M. Loey, "A deep learning model and machine learning methods for the classification of potential coronavirus treatments on a single human cell," Journal of Nano Particle Research, vol. 22, no. 10, pp. 1-13, 2020.

[17] A. Sampathkumar, S. Murugan, R. Rastogi, M. K. Mishra, S. Malathy et al., "Energy efficient acpi and jehdo mechanism for IoT device energy management in healthcare," in Internet of Things in Smart Technologies for Sustainable Urban Development, Cham: Springer, pp. 131-140, 2020.

[18] S. U. Amin, M. S. Hossain, G. Muhammad, M. Alhussein and M. D. A. Rahman, "Cognitive smart healthcare for pathology detection and monitoring," IEEE Access, vol. 7, pp. 10745-10753, 2019.

[19] K. Hameed, I. S. Bajwa, S. Ramzan, W. Anwar and A. Khan, "An intelligent IoT based healthcare system using fuzzy neural networks," Scientific Programming, vol. 2020, pp. 1-15, 2020.

[20] R. C. Chen, C. Dewi, S. W. Huang and R. E. Caraka, "Selecting critical features for data classification based on machine learning methods," Journal of Big Data, vol. 7, no. 52, pp. 1-26, 2020.

[21] J. Alghazo, G. Rathee, S. Gupta, M. T. Quasim, S. Murugan et al., "A secure multimedia processing through block chain in smart healthcare systems," ACM Transactions on Multimedia Computing Communications, and Applications (TOMM), vol. 1, pp. 1-12, 2020.

[22] M. Reyna, C. Josef, R. Jeter, S. Shashikumar, B. Moody et al., "Early prediction of sepsis from clinical data-the physionet computing in cardiology challenge 2019 (version 1.0.0)," PhysioNet. vol. 1, pp. 1-26, 2019. https://doi.org/10.13026/v64v-d857. 